\title{
Tract-based spatial statistics of diffusion tensor imaging in hereditary spastic paraplegia with thin corpus callosum reveals widespread white matter changes
}

\author{
Kader Karlı Oğuz, Eser Şanverdi, Arzu Has, Çağrı Temuçin, Sueda Türk, Katja Doerschner
}

\begin{abstract}
PURPOSE
We aimed to investigate white matter diffusivity abnormalities in hereditary spastic paraplegia with thin corpus callosum (HSP-TCC) patients in relation with electrophysiological findings.

MATERIALS AND METHODS

Brain magnetic resonance imaging (MRI) and diffusion tensor imaging were performed on four HSP-TCC patients and 15 age-matched healthy subjects. Voxel-wise statistical analysis of fractional anisotropy, axial diffusivity, radial diffusivity, and mean diffusivity maps were carried out using tract-based spatial statistics, and significantly affected voxels were labeled using a human white matter atlas. Conventional nerve conduction studies, cortical and spinal-root motor evoked potentials, and somatosensory evoked potentials were examined in three patients.

RESULTS

On MRI, all patients had a thin corpus callosum with mild T2 hyperintensity in the periventricular white matter. Compared to control subjects, we detected widespread significant decreases in fractional anisotropy, and increases in axial diffusivity, radial diffusivity, and mean diffusivity in structures including in the corpus callosum, motor, and non-motor white matter tracts in HSP-TCC patients. Several different regions showed significant reduction in axial diffusivity. Electrophysiological studies revealed prolonged central motor conduction times and reduced cortical motor evoked potentials and somatosensory evoked potentials amplitudes in all patients. One patient had low sural sensory nerve action potential suggestive of axonal neuropathy

\section{CONCLUSION}

Tract-based spatial statistics of diffusion tensor imaging revealed a more widespread involvement of white matter in HSP-TCC patients than has previously been detected by conventional MRI. This may explain the broad spectrum of electrophysiological and neurological abnormalities that complicate hereditary spastic paraplegia in these patients.
\end{abstract}

From the Department of Radiology (K.K.O. $\square$ karlioguz@yahoo. com, E.S.), Hacettepe University School of Medicine, Ankara, Turkey; National Magnetic Resonance Research Centre (UMRAM) (K.K.O., A.H., S.T., K.D.), Bilkent University, Ankara, Turkey; the Department of Neurology (Ç.T.), Hacettepe University School of Medicine, Ankara, Turkey.

Received 6 September 2012; revision requested 10 October 2012; revision received 15 October 2012; accepted 16 October 2012.

Published online 9 Jaunary 2013 DOI 10.5152/dir.2013.046
$\mathbf{H}$ ereditary spastic paraplegia (HSP) describes a group of neurodegenerative disorders that can either be pure (isolated HSP), with progressive spasticity, or complicated. HSP is classified as "complicated" when it co-occurs with other abnormalities such as mental and cognitive changes, optic atrophy, amyotrophy, ataxia, deafness, ichthyosis, and peripheral neuropathy $(1,2)$. In autosomal recessive HSP with thin corpus callosum (HSP-TCC), a form of complicated HSP, a patient typically has normal early motor and mental development followed by progressive spastic paraparesis, cognitive impairment, sensory disturbances, and ataxia during the second decade of life (3). First described by Iwabuchi et al. (4), HSP-TCC has been genetically linked to the SPG11 locus 15q13-15, although some heterogeneity has been described (5).

There are a few studies in the published literature describing the morphological, pathological, and imaging features of a limited number of HSP-TCC patients. The main feature of HSP-TCC seen on structural magnetic resonance imaging (MRI) is marked thinning of the corpus callosum; this is especially prominent in the genu and the body. In addition, a subtle, diffuse increase in the T2 signal intensity of the subcortical white matter (WM) and mild cerebellar and thalamic atrophy have also been variably reported $(1,6)$.

Diffusion tensor imaging (DTI) measures the anisotropic diffusion of water in WM tracts. In WM, diffusion is anisotropic, i.e. larger along the orientation of the axon and restricted in the cross-sectional direction. WM diffusion properties are influenced by structural factors such as the degree of myelination, the homogeneity of the orientation of fibers within the bundle, and/or damage. The dominant direction of a fiber bundle and the relative strength of diffusion in all directions can be estimated by fitting a tensor model to the diffusion data and by deriving different measures of diffusivity. These diffusivity measures include fractional anisotropy (FA), axial diffusivity (AD), radial diffusivity (RD), and mean diffusivity (MD). DTI also allows for the visualization of WM fasciculi in two and three dimensions as well as the detection of WM abnormalities before they are seen by structural MRI (7). Whole-brain analysis can be accomplished by tract-based spatial statistics (tract-based spatial statistics [TBSS], functional MRI of the brain [FMRIB], the FMRIB Software Library [FSL]) that enable voxel-wise analysis of multi-subject FA data (8).

Although studies of DTI performed on HSP patients have been published, only a few reports on a limited number of patients have described the WM abnormalities associated with $\operatorname{HSP}-T C C(1,3,9,10)$. None of these studies attempted to find correlations between DTI findings and functional abnormalities. The goal of this study was to quantify diffu- 
sion indices in order to characterize WM changes and then relate those changes with functional disturbances on electrophysiological studies.

\section{Materials and methods Subjects}

The local institutional review board approved this prospective study and all subjects signed informed consent forms before participation. Four patients with HSP-TCC (three males, one female) aged 18, 21, 28 and 18 years, (mean age, 21 years), respectively, and 15 healthy control subjects (mean age, 22 years; age range, 18-28 years) were included in the study. The HSP-TCC patients had normal motor and mental development before subsequently developing manifestations of spastic paraparesis $(n=4)$, peripheral neuropathy $(n=4)$, cerebellar ataxia $(n=2)$, and mild cognitive impairment $(n=2)$ at some point between the end of their first decade and early in their second decade of life. The mean duration of symptoms was seven years (Table 1 ). All patients met the diagnostic criteria for HSP-TCC (11) and had consanguineous parents. Three patients had a second-degree relative with similar clinical features; however, genetic studies could not be performed due to a lack of consent from the patients.

Healthy control subjects were volunteers who had no previous neurological, psychological, or systemic disease. All control subjects had normal results on brain MRI.

\section{Image acquisition}

Imaging studies were performed on a 1.5 Tesla MR scanner (Magnetom, Symphony TIM system, Siemens Medical Systems, Erlangen, Germany) equipped with an eight-channel head coil. Routine brain MRI pulse sequences included sagittal and axial T1-weighted spin-echo (SE) (TR/TE, 500/20 ms; matrix, $192 \times 256)$, axial and coronal T2-weighted fast SE (TR/ TE, 4000/100 ms; matrix, $192 \times 256$ ), and axial fast fluid-attenuated inversion-recovery (FLAIR) (TR/TE/TI, 9000/100/2100 ms; matrix, $192 \times 256$ ) images. All images had a $5 \mathrm{~mm}$ slice thickness with a 10\% interslice gap. No contrast material was administered to patients. A neuroradiologist (K.K.O.) and a neuroradiology fellow (E.S.) both evaluated MR images in consensus.

For midsagittal evaluation of the corpus callosum and an anatomical registration, three-dimensional sampling perfection with application-optimized contrasts using different flip angle evolutions FLAIR (TR/TE/TI, 5000/400/1800 ms; matrix, 218×256) imaging was obtained prior to the DTI sequence. DTI used a single-shot echo-planar imaging sequence and was performed in the axial plane with the following parameters: TR/TE, 5814/98 ms; maximum b factor, $1000 \mathrm{~s} / \mathrm{mm}^{2} ; 60$ independent directions; FOV, 230×230 mm; matrix, $128 \times 128 ; 25$ sections with $3 \mathrm{~mm}$ thickness without any intersection gap covering the cerebrum, cerebellum, and brain stem.

\section{Data processing and DTI}

Voxel-wise statistical analysis of the DTI data was carried out using TBSS implemented within the FSL 4.0 software package (Centre for FMRIB, Oxford University, Oxford, UK). Pre-processing of the diffusion-weighted data included head motion and eddy current correction and diffusion tensor fitting (FSL DTIFit). FA maps were registered and aligned to the average space as input for TBSS, and a thinned mean FA skeleton was computed. Then voxel-wise statistical analysis comparing $\mathrm{FA}, \mathrm{AD}, \mathrm{RD}$ and $\mathrm{MD}$ in control and HSP-TCC subjects was performed using an unpaired t test. The null distribution of the cluster-size statistic was built up over 500 permutations of group membership. The resulting Threshold-Free Cluster Enhancement output was corrected, and family-wise error-corrected maps were obtained with $P$ values less than 0.05. The WM structures with significant group differences in all diffusion metrics were extracted as regions of interest, registered and overlaid onto an anatomical template, and labeled using the "MR Imaging Atlas of Human White Matter" previously generated by Oishi et al. (12). FA, AD, RD, and $\mathrm{MD}$ values from the same regions of interests were recorded.

\section{Electrophysiological studies}

Three HSP-TCC patients underwent electrophysiological studies. Conventional nerve conduction studies were performed in upper and lower extremities. In motor evoked potential (MEP) studies, cortical MEPs were recorded at both tibialis anterior muscles by applying transcranial magnetic stimulation. Additionally, spinal-root MEPs were recorded bilaterally at tibialis anterior muscles by applying magnetic stimulation to motor roots over the fourth lumbar spinous processes. Central motor conduction time (CMCT) was calculated as the difference between the latency of cortical and spinal-root MEPs. Somatosensory evoked potentials (SEP) were studied bilaterally by stimulating the posterior tibial nerves and recording over the T12 spinal process and vertex. Central conduction time was calculated as the time between the latency of the spinal (N22) and cortical (P40) SEP potentials.

\section{Results}

\section{Brain structural MRI}

The corpus callosum was diffusely thin in all HSP-TCC patients (Fig. 1). All patients had either mild $(n=3)$ or moderate $(n=1)$ cerebral atrophy with a mildly increased $\mathrm{T} 2$ signal in the anterior (ears of lynx appearance) and posterior periventricular WM. We did not observe any atrophy of the cerebellum, thalami, or basal ganglia, nor did we observe any signal change in these structures.

\section{DTI}

The TBSS analysis revealed widespread WM alterations. Reduced FA and increased RD, AD, and MD (Fig. 2) dominated in the WM. The entire corpus callosum, thalami, internal and external capsules, anterior thalamic radiations, bilateral cingulum, fornices, superior longitudinal fasciculi, uncinate fasciculi, sagittal stratum, brain stem, and the cerebellum showed significantly lower FA in patients with HSP-TCC than in healthy subjects.

The RD was increased in all these areas except the corpus callosum (Fig. 2a). Furthermore, the corpus callosum showed mostly increased $\mathrm{AD}$ and $\mathrm{MD}$ as well as some small clusters of decreased $\mathrm{AD}$ in the genu and body. A few additional clusters with significantly reduced $\mathrm{AD}$ were also found in bilateral occipital WM, the anterior limb of the 

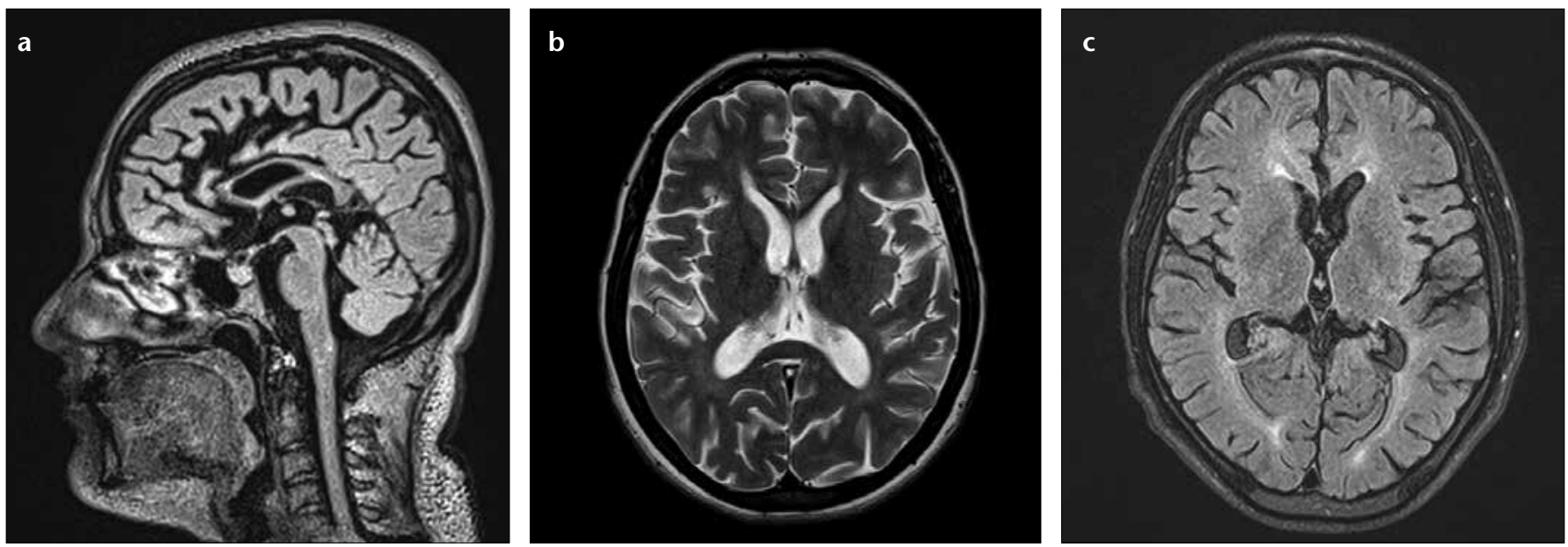

Figure 1. a-c. Sagittal FLAIR (a) and axial T2-weighted fast spin-echo (b) images of a 28-year-old patient show thinning of the corpus callosum. The thinning is more pronounced in the genu and body (a). Note periventricular frontal (ears of lynx appearance) and occipital white matter hyperintensity (b). Axial FLAIR image of another patient (c) also shows periventricular white matter hyperintensity.
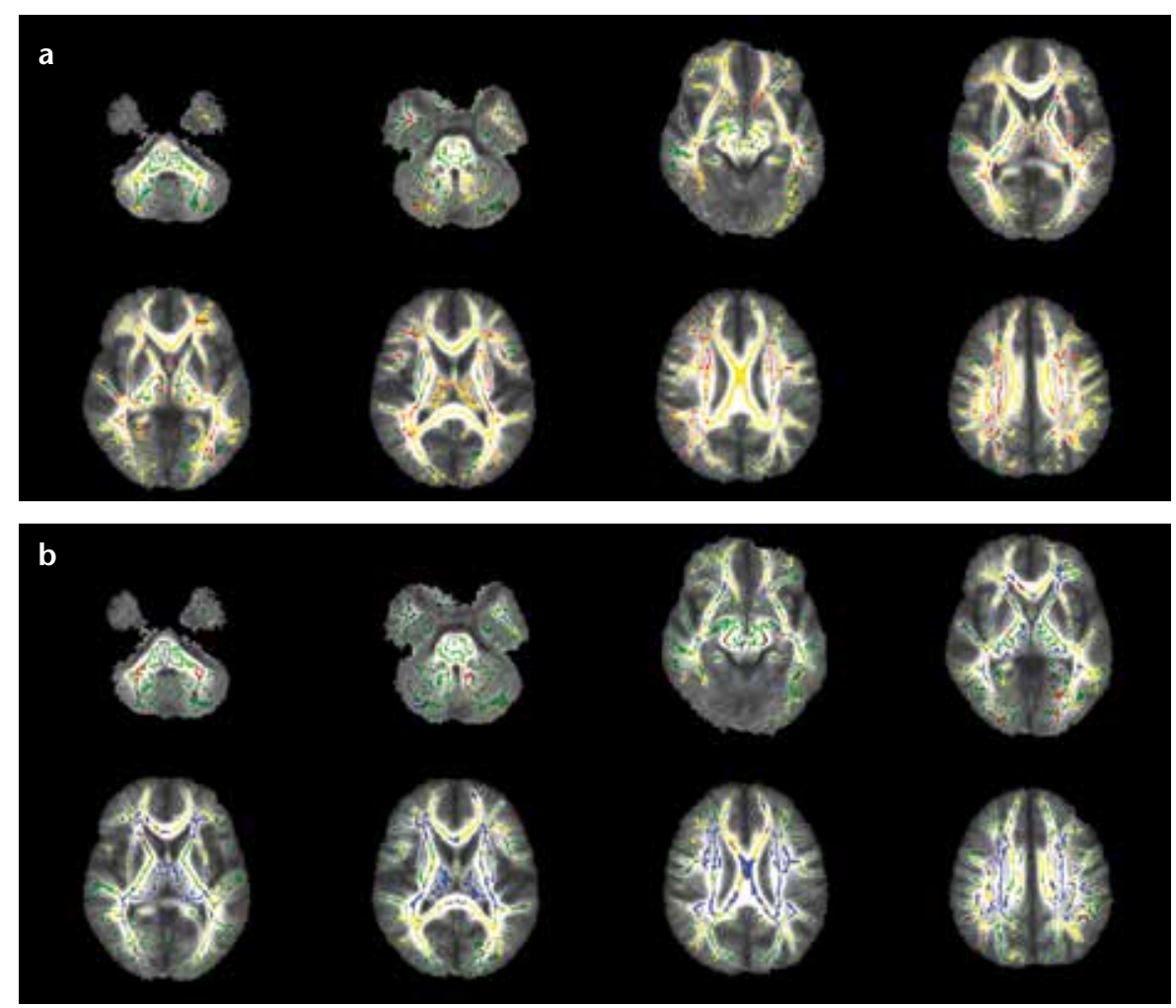

Figure 2. a, b. Family-wise error-corrected tract-based spatial statistics reveals regions of significantly reduced fractional anisotropy (a, yellow), increased radial diffusivity (a, red), increased mean diffusivity (b, yellow), decreased axial diffusivity (b, red), and increased axial diffusivity $(\mathbf{b}$, blue $)$ compared to control group $(P<0.05)$. The fractional anisotropy skeleton projected on the mean fractional anisotropy map is shown in green. Note that changes predominantly suggestive of demyelination are seen in the bilateral middle cerebellar peduncles and corticospinal tracts, in addition to widespread myelin and axonal abnormalities.

right internal capsule, the brain stem, and middle cerebellar peduncles (Fig. 2b). No FA increases or MD decreases were present. The diffusion measurements in regions of interests from significant clusters in patients and from corresponding areas in control subjects are presented in Table 2 .

\section{Electrophysiological studies}

The results of the electrophysiological assessments are summarized in
Table 3. Distal sensory axonal neuropathy was detected in one patient whose sural nerve sensory action potential amplitudes were decreased bilaterally. Furthermore, in one patient, cortical MEP response amplitudes were reduced, and CMCT was significantly prolonged bilaterally. However, there were only mild prolongations of CMCT in the other two patients. SEP revealed bilateral abnormalities in two patients while the other patient had normal values. In those two patients, cortical potential amplitudes were significantly reduced and central conduction time was significantly prolonged.

\section{Discussion}

In this study, we evaluated different diffusion indices in order to understand how WM changes correlate with functional changes in HSP-TCC patients. Consistent with previous studies, we observed thin corpus callosums and subtle WM T2 hyperintensities on structural MRI $(3,13)$. Although not an exact correlation, DTI provides invaluable information about the histopathological changes within the investigated brain parenchyma (7). The FA is an accepted measure of WM structural integrity, and decreases in the number or packing densities of axons, changes in their collinearity, or decreased orientational coherence of fiber tracts may lead to reduced $\mathrm{FA}$ (7). Whereas $\mathrm{AD}$ and $\mathrm{RD}$ measure the diffusion of water parallel to and perpendicular to WM fibers, respectively, MD gives a measurement of the average molecular motion independent of the 
Table 1. Demographic and clinical features of the patients

\begin{tabular}{|c|c|c|c|c|c|c|c|c|}
\hline Patient no & Age & Gender & $\begin{array}{c}\text { Age at disease } \\
\text { onset }\end{array}$ & $\begin{array}{c}\text { Spastic } \\
\text { paraplegia }\end{array}$ & $\begin{array}{l}\text { Peripheral } \\
\text { neuropathy }\end{array}$ & $\begin{array}{l}\text { Cognitive } \\
\text { impairment }\end{array}$ & Ataxia & Parkinsonism \\
\hline 1 & 18 years & Male & 10 years & Yes & Yes & Mild & Yes & Yes \\
\hline 3 & 28 years & Male & 21 years & Yes & Yes & No & Yes & No \\
\hline 4 & 18 years & Female & 12 years & Yes & Yes & No & No & No \\
\hline
\end{tabular}

Table 2. FA, MD, AD, and RD values from significant clusters of the patients and corresponding areas of healthy control subjects

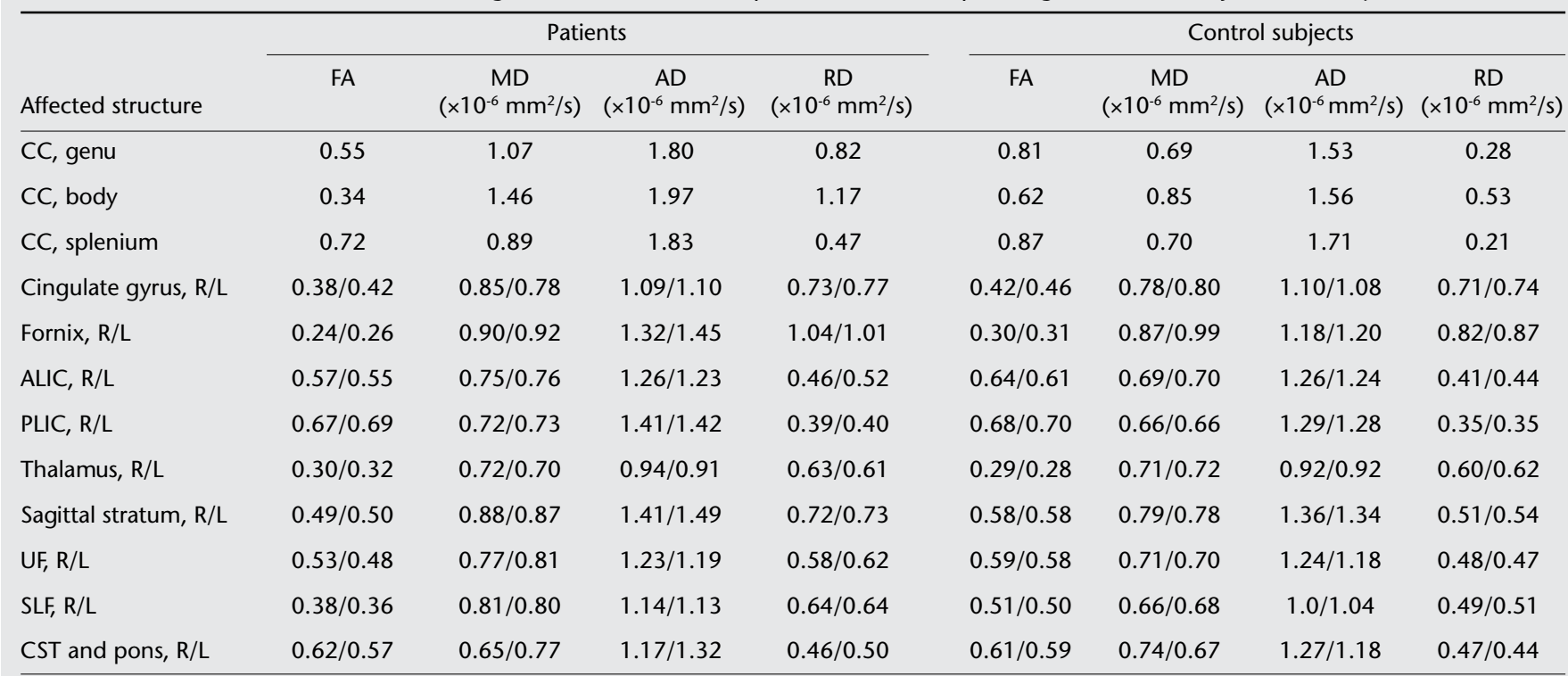

$\mathrm{AD}$, axial diffusivity; ALIC, anterior limb of internal capsule; CC, corpus callosum; CST, corticospinal tract; FA, fractional anisotropy; MD, mean diffusivity; PLIC, posterior limb of internal capsule; R, right; L, left; RD, radial diffusivity; SLF, superior longitudinal fasciculus; UF, uncinate fasciculus.

Table 3. Summary of electrophysiological findings

\begin{tabular}{llll}
\hline Patient no & NCS & MEP & SEP \\
\hline 1 & Low sural SNAP & Mild CMCT prolongation & Normal \\
2 & - & $\begin{array}{l}\text { Reduced cortical MEP } \\
\text { amplitude and significant } \\
\text { CMCT prolongation }\end{array}$ & $\begin{array}{l}\text { Reduced cortical SEP } \\
\text { amplitudes and significant }\end{array}$ \\
& \multirow{2}{*}{$\begin{array}{l}\text { CCT prolongation } \\
3\end{array}$} & $\begin{array}{l}\text { Reduced cortical MEP } \\
\text { amplitude and significant }\end{array}$ & $\begin{array}{l}\text { Reduced cortical SEP } \\
\text { amplitudes and significant }\end{array}$ \\
& CMCT prolongation & CCT prolongation
\end{tabular}

direction of diffusion. Instead, cellular size, compartmental fluid imbalance, and degradation products all affect MD $(14,15)$. A reduction in FA is usually accompanied by an increase in $\mathrm{RD}$, reflecting a de/dysmyelinating process. $\mathrm{AD}$ has previously been shown to be a primary measure of axonal injury (16).

In our study, TBSS analysis revealed widespread reductions in FA and increases in MD, RD, and AD in HSP-TCC patients, with the exception of a few significant clusters of decreased AD. These changes, suggestive of both axonal and myelin damage, were observed in the projection, commissural and association fibers of the brain. Involvement of the parahippocampal gyri and connected structures such as fornices, cingulum, and thalami was striking and may partly explain the cognitive impairment in patients with HSP-TCC. França et al. (10) recently demonstrated involvement of the WM beyond the corpus callosum and motor pathways in HSP-TCC patients. However, they also observed increased $\mathrm{AD}$ in the thalamus and frontal WM, but not in the posterior regions, whereas we observed reduced $\mathrm{AD}$ in the corresponding occipital WM. We found that the splenium of the corpus callosum, which showed no remarkable DTI abnormality in the study by França et al. (10), was affected in addition to more anterior parts of the corpus callosum. Along with the lack of any appreciable RD change on the TBSS map, these findings suggest that main histopathological alteration in HSPTCC patients may be axonal damage rather than demyelination in the corpus callosum. Based on a postmortem pathological examination, Wakaba- 
yashi et al. (17) also proposed that HSPTCC correlates with maldevelopment of the corpus callosum and substantia nigra with subtle gliosis as well as well-preserved myelin associated with a severely attenuated corpus callosum. However, the general understanding is that both maldevelopmental and neurodegenerative processes occur throughout the course of HSP-TCC (17-19).

One previous application of DTI in motor neuron diseases included 24 pure HSP patients and reported significant alterations in motor pathways and projections to the limbic system and corpus callosum (20). In our analysis, patients with HSP-TCC had additional widespread alterations in other areas, including the brain stem, cerebellum and anterior thalamic radiations, thalami, superior longitudinal fasciculi, uncinate fasciculi, and sagittal stratum.

In our patient group, we did not observe any abnormalities in the corona radiata, internal capsules, brain stem, or cerebellum on T2-weighted images. However, our results suggest anisotropy changes along the cortico-spinal tract. This is in agreement with electrophysiological findings showing various degrees of conduction disturbance in the CMCT of three patients.

In addition to MEPs that indicate conduction disturbance at the corticospinal motor pathways rostral to L4 spinal segment, sensory neuropathy of an axonal nature has previously been reported in HSP; this was also observed in one of our patients (21). Furthermore, SEP revealed a bilateral abnormality at the dorsal column-medial lemniscal sensory pathways in two patients, consistent with previous reports $(22,23)$. MEP and SEP abnormalities in HSP patients have generally been attributed to spinal cord involvement $(22,23)$. However, the abnormalities at the internal and external capsules, thalamic projections and brain stem that we detected using TBSS-DTI analysis may also contribute to MEP and SEP abnormalities. Further DTI analysis of the spinal cord in HSP-TCC patients may advance the understanding of the structural abnormalities underlying electrophysiological findings associated with this condition.

Our data supports the involvement of the thalamus in the neurodegenerative progression of HSP-TCC even though its volume and signal on brain MRI is normal. This finding agrees with previous single photon emission computed tomography using N-isopropyl-(iodine-123)p-iodoamphetamine, pathological, and MRI morphometry studies showing reduced blood flow, neuronal loss, gliosis, and atrophy in the thalamus $(13,18,10,24)$.

The primary limitation of this study is the lack of genetic analysis due to patients' reluctance. However, the patients' clinical history, MRI features, and electrophysiological studies, which were further supported by previously published clinical criteria, enabled the diagnosis of HSP-TCC. Our patient group was small but relatively homogenous with respect to the duration and severity of the disease. It is possible, though, that the extent and severity of DTI abnormalities could change depending on the duration and severity of the disease and in parallel with the changes in the WM (17). Given the disorder's rarity and the scarcity of reports in the literature, we feel that our results support previous histopathological reports proposing that the structural changes associated with HSP-TCC go far beyond just the corpus callosum and motor pathways.

In conclusion, our study provides much needed insight into the widespread reduced WM integrity in HSPTCC patients, which appears to be substantially more pronounced than can be seen in structural MRI. Furthermore, the abnormal motor and somatosensory functions seen in electrophysiological studies may provide a correlate for the widespread tissue alterations observed by DTI. Future longitudinal studies with increased patient enrollment and a spectrum of phenotypes will be needed to shed further light on the pathophysiology, clinical correlates, and course of HSP-TCC.

\section{Conflict of interest disclosure}

The authors declared no conflicts of interest.

\section{References}

1. Chen Q, Lui S, Wang JG, et al. Diffusion tensor imaging of two unrelated Chinese men with hereditary spastic paraplegia associated with thin corpus callosum. Neurosci Lett 2008; 441:21-24. [CrossRef]

2. Fink JK. Hereditary spastic paraplegia. Neurol Clin 2002; 20:711-726. [CrossRef]
3. Dreha-Kulaczewski S, Dechent P, Helms $G$, et al. Cerebral metabolic and structural alterations in hereditary spastic paraplegia with thin corpus callosum assessed by MRS and DTI. Neuroradiology 2006; 48:893-898. [CrossRef]

4. Iwabuchi K, Yagishita S, Amano N, et al. A new type of complicated form of hereditary spastic paraplegia showing mental deterioration, quadriplegia with muscular atrophy, sensory disturbance, extrapyramidal disorders, and epilepsy. Rinsho Shinkeigaku 1991; 31:945-952.

5. Stevanin G, Montagna G, Azzedine H, et al. Spastic paraplegia with thin corpus callosum: description of 20 new families, refinement of the SPG11 locus, candidate gene analysis and evidence of genetic heterogeneity. Neurogenetics 2006; 7:149-156. [CrossRef]

6. França MC Jr, D'Abreu A, Maurer-Morelli $\mathrm{CV}$, et al. Prospective neuroimaging study in hereditary spastic paraplegia with thin corpus callosum. Mov Disord 2007; 22:1556-1562. [CrossRef]

7. Le Bihan D. Looking into the functional architecture of the brain with diffusion MRI. Nat Rev Neurosci 2003; 4:469-480. [CrossRef]

8. Smith SM, Jenkinson M, Johansen-Berg H, et al. Tract-based spatial statistics: voxelwise analysis of multi-subject diffusion data. Neuroimage 2006; 31:1487-1505. [CrossRef]

9. Ota M, Sato N, Saitoh Y, Endo F, Murata M, Asada T. Diffusion tensor imaging in familial spastic paraplegia with mental impairment and thin corpus callosum. Magn Reson Med Sci 2008; 7:163-167. [CrossRef]

10. França MC Jr, Yasuda CL, Pereira FR, et al. White and grey matter abnormalities in patients with SPG11 mutations. J Neurol Neurosurg Psychiatry 2012; 83:828-833. [CrossRef]

11. Nakamura A, Izumi $K$, Umehara $F$, et al. Familial spastic paraplegia with mental impairment and thin corpus callosum. J Neurol Sci 1995; 131:35-42. [CrossRef]

12. Oishi K, Andreia F, van Zijl PCM, Mori S. MRI atlas of human white matter. 2nd ed. Oxford: Elsevier, 2011.

13. Okubo S, Ueda M, Kamiya T, et al. Neurological and neuroradiological progression in hereditary spastic paraplegia with a thin corpus callosum. Acta Neurol Scand 2000; 102:196-199. [CrossRef]

14. Beaulieu C, Allen PS. Determinants of anisotropic water diffusion in nerves. Magn Reson Med 1994; 31:394-400. [CrossRef]

15. Pierpaoli C, Jezzard P, Basser PJ, Barnett A, Di Chiro G. Diffusion tensor MR imaging of the human brain. Radiology 1996; 201:637-648.

16. Budde MD, Xie M, Cross AH, Song SK. Axial diffusivity is the primary correlate of axonal injury in the experimental autoimmune encephalomyelitis spinal cord: a quantitative pixelwise analysis. J Neurosci 2009; 29:2805-2813. [CrossRef]

17. Wakabayashi K, Kobayashi $\mathrm{H}$, Kawasaki $\mathrm{S}$, et al. Autosomal recessive spastic paraplegia with hypoplastic corpus callosum, multisystem degeneration and ubiquitinated eosinophilic granules. Acta Neuropathol 2001; 101:69-73. 
18. Kuru S, Sakai M, Konagaya M, et al. Autopsy case of hereditary spastic paraplegia with thin corpus callosum showing severe gliosis in the cerebral white matter. Neuropathology 2005; 25:346-352. [CrossRef]

19. Kato S, Hirano A, Suenaga T, et al. Ubiquitinated eosinophilic granules in the inferior olivary nucleus. Neuropathol Appl Neurobiol 1990; 16:135-139. [CrossRef]

20. Unrath A, Müller HP, Riecker A, et al. Whole brain-based analysis of regional white matter tract alterations in rare motor neuron diseases by diffusion tensor imaging. Hum Brain Mapp 2010; 31:1727-1740.
21. Schady W, Smith CM. Sensory neuropathy in hereditary spastic paraplegia. J Neurol Neurosurg Psychiatry 1994; 57:693-698. [CrossRef]

22. Chady W, Dick JP, Sheard A, et al. Central motor conduction studies in hereditary spastic paraplegia. Central motor conduction studies in hereditary spastic paraplegia J Neurol Neurosurg Psychiatry 1991; 54:775-779.

23. Bruyn RP, van Dijk JG, Scheltens P, et al. Clinically silent dysfunction of dorsal columns and dorsal spinocerebellar tracts in hereditary spastic paraparesis. J Neurol Sci 1994; 125:206-211. [CrossRef]
24. Ohnishi J, Tomoda Y, Yokoyama K. Neuroradiological findings in hereditary spastic paraplegia with a thin corpus callosum. Acta Neurol Scand 2001; 104:191-192. [CrossRef] 